Research Article

\title{
The Evolution of the Cephalometric Superimposition Techniques from the Beginning to the Digital Era: A Brief Descriptive Review
}

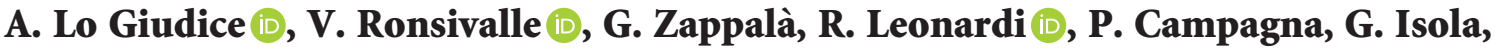 \\ and G. Palazzo \\ Department of General Surgery and Surgical-Medical Specialties, School of Dentistry, University of Catania, Via S. Sofia 78, \\ Catania 95124, Italy \\ Correspondence should be addressed to V. Ronsivalle; vincenzo.ronsivalle@hotmail.it
}

Received 19 November 2020; Revised 13 February 2021; Accepted 2 April 2021; Published 24 April 2021

Academic Editor: Boonlert Kukiattrakoon

Copyright ( 2021 A. Lo Giudice et al. This is an open access article distributed under the Creative Commons Attribution License, which permits unrestricted use, distribution, and reproduction in any medium, provided the original work is properly cited.

\begin{abstract}
Superimposition of craniofacial structures from radiographic examination has been always used for assessing changes in the maxillamandibular complexes, especially for the evaluation of potential changes occurring during growth as well as after orthodontic treatment and/or maxillofacial surgery. However, the availability of cone beam computed tomography (CBCT) and the recent advancement in 3D imaging have allowed the development of specific techniques for the registration and superimposition of virtual three-dimensional anatomical structures, improving the diagnosis and treatment plan strategies. In the present paper, it will be discussed the evolution of superimposition techniques from the beginning (2D) to the newest 3D approach, describing the most used methods and their main advantages and disadvantages, focusing primarily on accuracy and reproducibility of each technique.
\end{abstract}

\section{Introduction}

2Cephalometric superimposition is an analysis of lateral, frontal, or three-dimensional (3D) cephalograms of the same patient taken at different times. Since 1955, when Bjork [1] introduced this technique, cephalometric superimpositions gained growing importance due to the possibility to evaluate the patient's growth pattern between different ages, to evaluate changes in dentoalveolar and basal relationships after a course of orthodontic or surgical treatment, and to differentiate the changes due to growth and treatment [2].

In fact, superimpositions provide an overall assessment of the growth and treatment changes of the facial structures evaluating the amount and direction of maxillary and mandibular growth or displacement, changes in maxillarymandibular relationship, and relative changes in soft tissue integument (nose, lips, and chin) and provide information on the overall displacement of the teeth [3].

There are different methods to superimpose two or more $\mathrm{X}$-ray-derived datasets. Such methods can be classified in two main diagnostic approaches that have followed the enhancement of diagnostic imaging, i.e., two-dimensional (2D) and three-dimensional (3D) image techniques.

The early traditional method is a bidimensional (2D) evaluation based on the comparison of linear and angular measurements on serial superimpositions from cephalograms that have been taken at different times to evaluate the effect of growth or treatment. In this respect, tracings of the head films must be superimposed on relatively stable landmarks least affected by the growth, in order to be accurate and reproducible. Thus, superimpositions must be taken under identical conditions of magnification, head position, and radiological exposure, and tracing of the superimpositions must accurately locate the outlines or the relevant structures, eliminating confusing, unusable details.

Since lateral cephalogram is routinely used for the assessment of maxillofacial skeletal pattern as well as for the assessment of pre-post treatment changes, $2 \mathrm{D}$ cephalometric is still useful for orthodontists. However, conventional superimposition involves the representation of $3 \mathrm{D}$ complex 
structures, like the craniofacial complex, on a 2D flat X-ray film, with intrinsic limitation. Furthermore, 2D analysis has different drawbacks such as image distortion and magnification and difficulty in landmark identification due to the overlapping of the anatomical structures [4-7].

In order to overcome these disadvantages, nowadays, clinicians can refer to 3D imaging technology. In this respect, cone beam computed tomography (СВCT) represents the three-dimensional (3D) imaging method of choice in oral and maxillofacial fields [8]. Although multislice computed tomography (MSCT) presents higher resolution and higher contrast-to-noise ratio $[9,10]$, CBCT scans offer adequate images for diagnostic purpose, at lower risk of patients' radiation exposure [11-14]. Also, CBCT scans allow the rendering of anatomical dataset into a $3 \mathrm{D}$ anatomical model that could be used for linear, volumetric, and surface measurements, allowing a detailed analysis of the maxillofacial structures without magnification and distortion errors [12]. In particular, 3D rendering of maxilla and mandibular jaws is extremely useful for preoperative surgical planning and postoperative follow-up evaluation in orthognathic surgery and for the analysis of morphological, dimensional, and positional changes before and after orthodontic therapies $[13,14]$.

In the last years, a growing number of 3D superimposition techniques have been proposed by different authors using landmark-, surface-, or voxel-based registration. Each of these techniques allows the superimposition of consecutive CBCT-derived 3D models of the same patient in order to study the displacement and growth of the craniofacial complex, the facial asymmetries $[15,16]$, and the changes after an orthodontic or surgical treatment [17-22]. In the present paper, it will be discussed the evolution of superimposition techniques from the beginning (2D) to the newest 3D approach, describing the most used methods and their main advantages and disadvantages, focusing primarily on accuracy and reproducibility of each technique.

\section{Materials and Methods}

In this section, we provided and discussed a detailed description of the common methodologies used to perform superimposition of the craniofacial structures, according to the best available scientific contributions in orthodontic literature.

2.1. 2D Analysis. Cranial base structures have been used for superimpositions since both the neurocranium and its related cranial base achieve most of their growth potential at a relatively early age. Therefore, this part of the cranium is considered to be relatively stable. Several cranial axes were proposed in the past such as basion horizontal, broadbent triangle, basion-nasion plane, and sella-nasion line [23].

The areas that have to be evaluated include the changes in overall face, changes in the maxilla and its dentition, changes in the mandible and its dentition, amount and direction of condylar growth, and mandibular rotation.
Based on the American Board of Orthodontics color codes are used to facilitate identification of consecutive cephalograms (Figure 1):

(i) Pretreatment-black

(ii) Progress-blue

(iii) End of treatment-red

(iv) Retention-green

Although these methods are easy to use, they have some disadvantages since they incorporate areas of the cranial base that may still change during growth stage such as the spheno-occipital synchondrosis or area of bone remodeling such as those recognizable by cephalometric points Nasion and Sella. Moreover, the position of the basion is influenced by the remodeling process on the surface of the clivus and on the anterior border of the foramen magnum and by the displacement of occipital bone due to growth at sphenooccipital synchondrosis [24].

Based on these considerations, Planché [25] created the best fit method of anterior cranial base identifying various bony surfaces in the anterior cranial base that are suitable for accurate superimpositions. They include the anterior wall of sella turcica, the contour of cribriform plate of ethmoid bone, details in trabecular system in ethmoid cells, median border of orbital roof, and plane of sphenoid bone.

Moreover, superimposition of maxillary and mandibular jaws was used to allow the evaluation of changes of specific bone structures such as the maxilla and the mandible.

The purpose of maxillary superimposition is to evaluate the movement of maxillary teeth in relation to the basal parts of maxilla. In this respect, some methods used the palatal plane [25] such as

(i) Superimposition along the palatal plane registered at ANS [26-30]

(ii) Superimposition on the nasal floor with films registered at anterior surface of maxilla [31, 32]

(iii) Superimposition along the palatal plane registered at pterygomaxillary fissure [27]

These methods are compromised because of the remodeling of the palatal shelves and the fact that the hard palate undergoes continuous resorption on its nasal surface and apposition on the oral side, making most of these methods of superimposition unsatisfactory. Furthermore, both ANS and PNS undergo significant anteroposterior remodeling.

Other methods superimpose on other structures such as

(i) The outline of infratemporal fossa and posterior portion of hard palate [33]

(ii) Superimposition registering the maxilla on the common Pterygomaxillary, maintaining the basion horizontal relationship [34]

(iii) Superimposition on the best fit of internal palatal structures [30] 


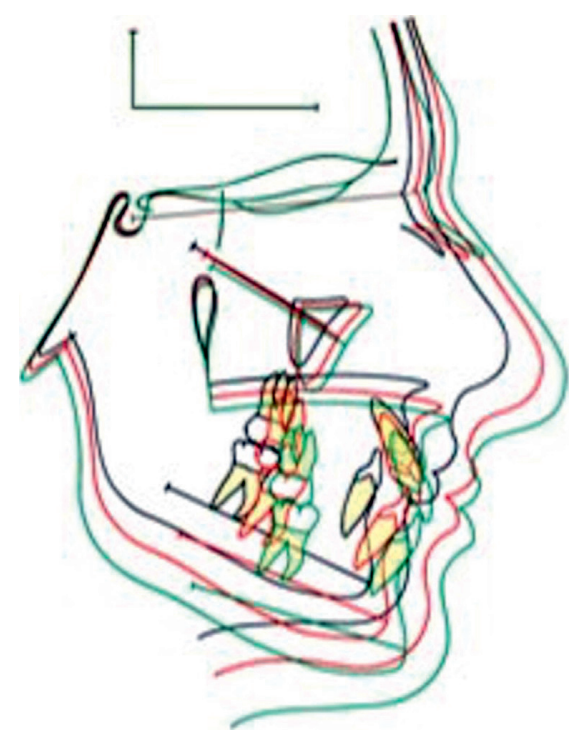

Figure 1: Example of 2D superimposition of cranial base structures.

(iv) Superimposition on metallic implants on the anterior surface of the zygomatic process of the maxilla [35]

Similarly to the maxilla, mandibular areas can be superimposed in order to evaluate the movement of mandibular teeth in relation to the basal parts of the mandible.

There are different methods that use various landmarks such as

(i) Superimposition on the lower border of the mandible and on the inner table of symphysis [28]

(ii) Superimposition on the mandibular plane

(iii) Superimposition on anterior contour of the chin, the inner contour of the cortical plates at the inferior border of the symphysis and trabecular structure in the lower part of symphysis, the contour of mandibular canal, and the lower contour of mineralized molar germ (Figure 2)

2.2. 3D Analysis. The introduction of CBCT technology and the possibility to render a volumetric patient's dataset in a $3 \mathrm{D}$ virtual model have increased a growing interest by the scientific community in developing different methods to study and analyze the morphology and changes of the craniofacial complex using $3 \mathrm{D}$ superimposition methods. The main two technologies used to superimpose 3D images are surface-based registration and voxel-based registration. These techniques have introduced the possibility to superimpose two or more consecutive CBCT-derived $3 \mathrm{D}$ models of the same patient in order to study the growth of the craniofacial complex, to evaluate surgical or orthodontic treatment outcomes, to analyze the facial asymmetries, or to plan a surgical treatment $[15,16]$. 3D registration techniques have different advantages with respect to $2 \mathrm{D}$ superimpositions. First of all, they well overcome the magnification, distortion, and landmark

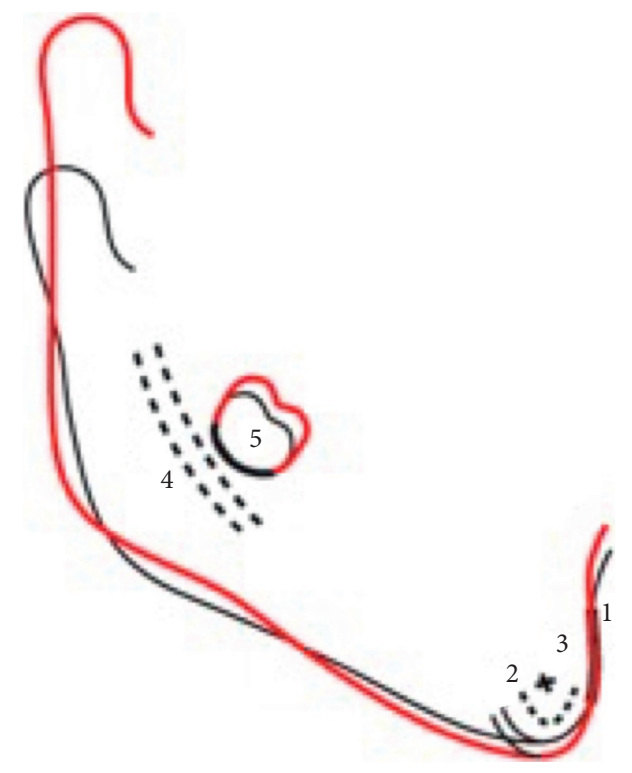

FIgURE 2: Example of 2D mandibular superimposition.

identification errors that frequently occur in 2D cephalometry [7]. Furthermore, the possibility to render a volumetric dataset in a 3D model of different anatomical structures (i.e., mandible, maxillary bone, and cranial base) allows to finely analyze the morphological changes of the whole craniofacial complex or of a specific anatomical area, giving the clinician a wider range of information with respect to traditional cephalometry. In addition to $2 \mathrm{D}$ superimpositions techniques, $3 \mathrm{D}$ registration methods permit to study the displacement of anatomical point in each direction of the space once a correct orientation of the head is preliminary achieved. Furthermore, using a 3D superimposition approach, it is possible to calculate the Euclidean distances between the surface of the registered $3 \mathrm{D}$ models and to highlight this deviation values in a colorimetric map using an iterative closest point evaluation method [36, 37]. As reported in literature, a good superimposition method should be able to register precisely and aid in understanding the changes as a result of growth and/ or treatment relative to the structure of reference. According to previous studies, 3D superimposition techniques provide not only better accuracy and reliability with respect to $2 \mathrm{D}$ traditional techniques but also a better understanding of the morphological changes of the superimposed structures.

The first method described in the literature for superimposition of $3 \mathrm{D}$ datasets is the surface-based registration (SBR). It was proposed by Hajeer et al. [36] in order to study the facial asymmetry before and after orthognathic surgery. This technique uses the surface of two or more segmented structures to superimpose them and requires a high-quality surface of 3D models for an accurate superimposition. It is also called "best fit" algorithm due to the iterative process (Iterative Closest Point (ICP) algorithm) which minimizes the surface distance between the two surfaces previously manually approximated (Figure 3). SBR calculated an estimation of the optimal rotational and translational 

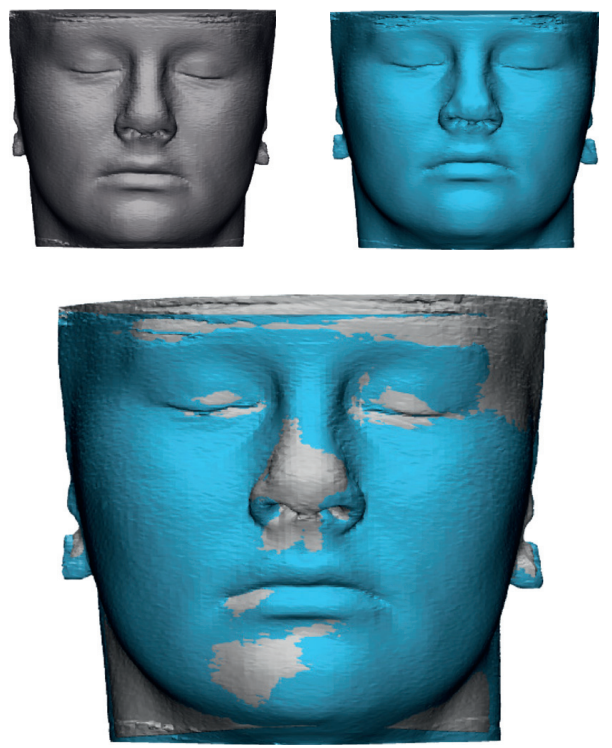

FIgURE 3: Example of surface-based superimposition of CBCT-derived patient's facial soft tissues before and after orthodontic treatment with maxillary expander.
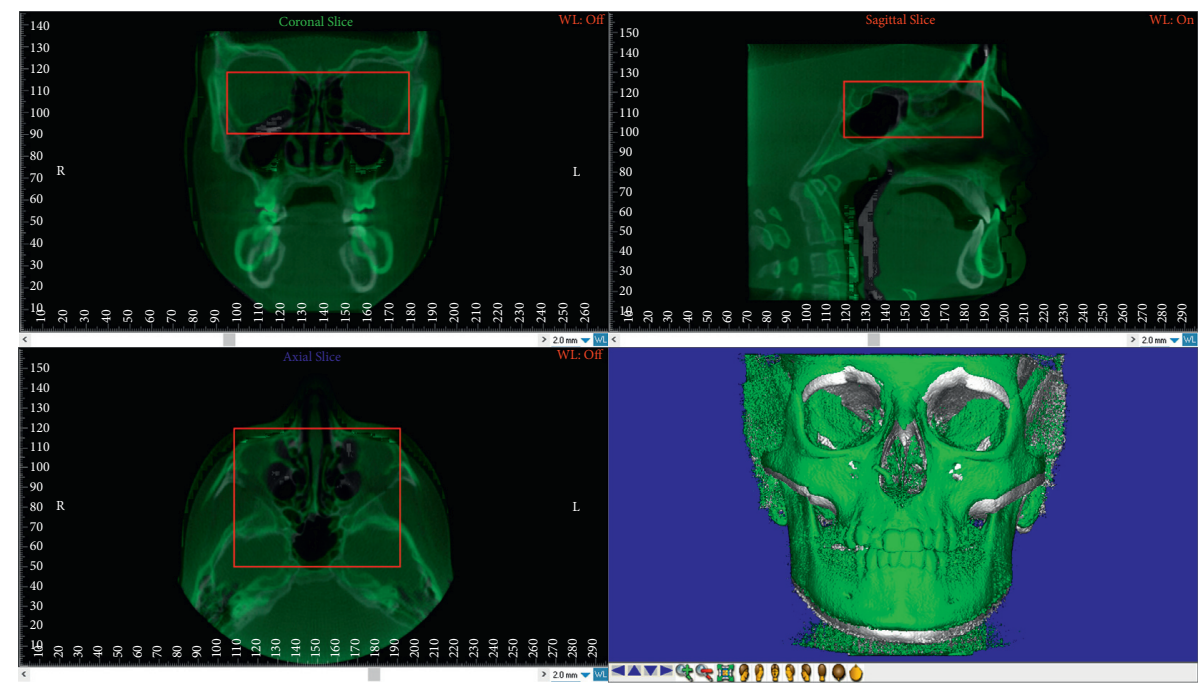

Figure 4: Voxel-based superimposition: selection of the Volume of Interest (VOI) on the cranial base structure of T0-CBCT (before treatment) and registration of the T1-CBCT (posttreatment).

movements between the surface of $3 \mathrm{D}$ models by minimizing the mean square distance between the models' mesh points. This distance is measured between a specified percentage of the points randomly selected on one 3D mesh and the corresponding $3 \mathrm{D}$ surface mesh. This technique, firstly proposed to study facial asymmetry, has been used by different authors in order to study the asymmetry and morphology of different anatomical districts such as the palatal vault, the mandible, the glenoid fossa, and other facial structures [37].

The voxel-based registration (VBR) is an automatic superimposition technique firstly proposed by Planché et al. $[25,38]$ involving the matching between the greyscale values of the voxels within the selected Volume of Interest (VOI) of two or more volumetric datasets (i.e., CBCT and CT) (Figures 4 and 5). One of the most important advantages of this technique is that, unlike landmark- and surface-based registration, it does not depend on the accuracy of landmark identification or on errors in the surface segmentation process. In fact, it is a completely automated registration method which avoids observer-dependent errors. Nowadays, there are two software applications available for performing voxel-based registration, i.e., Dolphin and Slicer 3D, both of them showing both high accuracy and reliability $[17,18,39,40]$. This technique was firstly proposed for identifying overall treatment outcomes and different patterns of remodeling following orthognathic surgery in non-growing subjects using as reference structure the anterior cranial base $[18,38]$. Afterwards, it was used for 


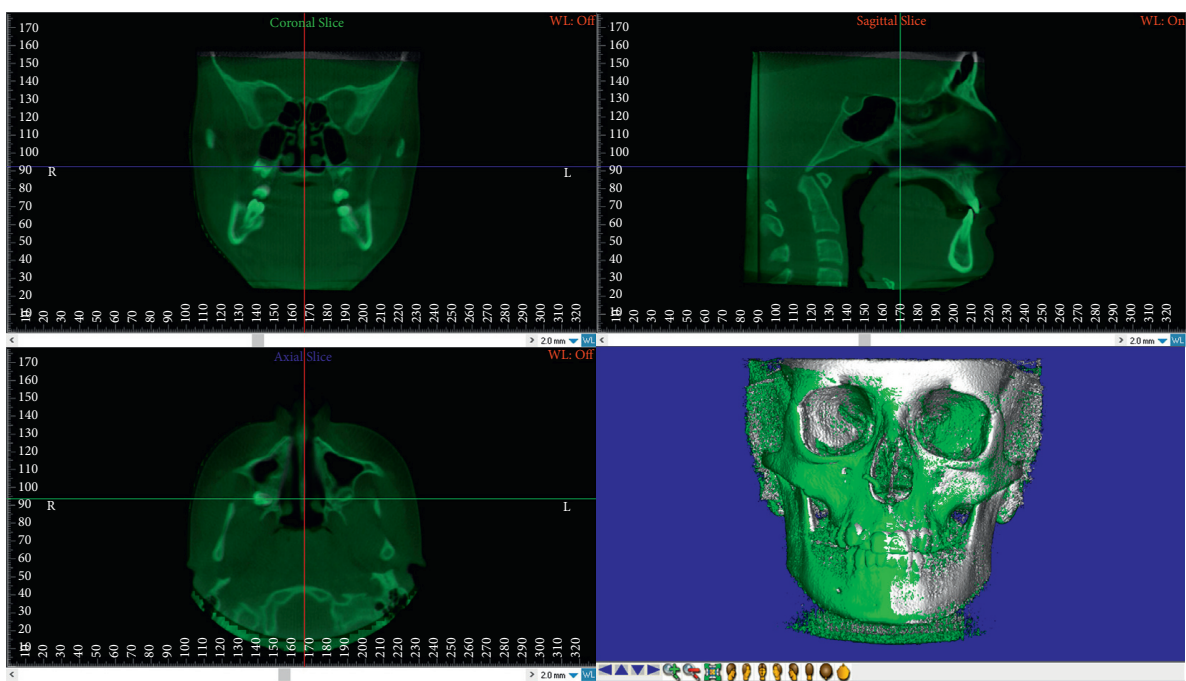

FIGURE 5: Voxel-based superimposition completed.

assessing the facial overall treatment outcomes (i.e., maxillary and/or mandibular displacement) in growing subjects [41] and proposed for regional registration on maxillary or mandibular bone by different authors. Ruellas et al. [17] compared different mandibular reference regions for analyzing the changes in dental arches and/or condyle/rami complex due to orthodontic treatment. Koerich et al. [22] described a voxelbased registration for maxillary and mandibular region as alternative to the anterior cranial base. Nada et al. [42] compared the accuracy and reliability of voxel-based registration using the zygomatic arch instead of the anterior cranial base in smaller FOV CBCT, concluding that the zygomatic arch can be used as stable structure. Previous studies [43, 44] compared landmark- and voxel-based registration in order to evaluate changes in mandibular condyles morphology after maxillofacial surgery [45].

Based on studies comparing the three registration methods previously cited, landmark-based superimposition is the least reliable due to the need of manual landmark selection by the operator and the lack of precise definition of $3 \mathrm{D}$ coordinates of cephalometric landmarks. Surfacebased registration and voxel-based registration have been widely validated, the voxel-based registration being associated with less variability in terms of accuracy and reliability. In this regard, surface-based registration preliminary requires a precise segmentation of the investigated structures, which can introduce biases in the superimposition method. It was also suggested that initial approximation of the images is an important step to reduce registration time of the software and improve the precision of the superimposition.

\section{Conclusions}

Considering the continuous update in 3D imaging, clinicians must get familiar with these new technologies that have opened new scenario in the diagnosis and treatment plan of patients involved in orthodontic and maxillofacial therapies.

\section{Data Availability}

The data used to support the findings of this study are available from the corresponding author upon request.

\section{Conflicts of Interest}

The authors declare that there are no conflicts of interest regarding the publication of this paper.

\section{References}

[1] A. Björk, "Facial growth in man, studied with the aid of metallic implants," Acta Odontologica Scandinavica, vol. 13, no. 1, pp. 9-34, 1955

[2] R. M. Ricketts, "A four-step method to distinguish orthodontic changes from natural growth," Journal of Clinical Orthodontics: JCO, vol. 9, no. 4, pp. 208-228, 1975.

[3] J. Ghafari, F. E. Engel, and L. L. Laster, "Cephalometric superimposition on the cranial base: a review and a comparison of four methods," American Journal of Orthodontics and Dentofacial Orthopedics, vol. 91, no. 5, pp. 403-413, 1987.

[4] A. Sandham, "Repeatability of head posture recordings from lateral cephalometric radiographs," British Journal of Orthodontics, vol. 15, no. 3, pp. 157-162, 1988.

[5] Y. J. Yoon, D. H. Kim, P. S. Yu, H. J. Kim, E. H. Choi, and K. W. Kim, "Effect of head rotation on posteroanterior cephalometric radiographs," The Angle Orthodontist, vol. 72, no. 1, pp. 36-42, 2002.

[6] A. M. Cohen, "Uncertainty in cephalometrics," British Journal of Orthodontics, vol. 11, no. 1, pp. 44-48, 1984.

[7] W. J. B. Houston, R. E. Maher, D. McElroy, and M. Sherriff, "Sources of error in measurements from cephalometric radiographs," The European Journal of Orthodontics, vol. 8, no. 3, pp. 149-151, 1986.

[8] W. C. Scarfe, A. G. Farman, and P. Sukovic, "Clinical applications of cone-beam computed tomography in dental practise," Journal of the Canadian Dental Association, vol. 72, pp. 75-80, 2006.

[9] W. P. Engelbrecht, Z. Fourie, J. Damstra, P. O. Gerrits, and $Y$. Ren, "The influence of the segmentation process on $3 \mathrm{D}$ measurements from cone beam computed tomography- 
derived surface models," Clinical Oral Investigations, vol. 17, no. 8, pp. 1919-1927, 2013.

[10] L. F. P. Nicolielo, J. Van Dessel, E. Shaheen et al., "Validation of a novel imaging approach using multi-slice CT and conebeam CT to follow-up on condylar remodeling after bimaxillary surgery," International Journal of Oral Science, vol. 9, no. 3, pp. 139-144, 2017.

[11] T. Xi, B. Van Loon, P. Fudalej, S. Bergé, G. Swennen, and T. Maal, "Validation of a novel semi-automated method for three-dimensional surface rendering of condyles using cone beam computed tomography data," International Journal of Oral and Maxillofacial Surgery, vol. 42, no. 8, pp. 1023-1029, 2013.

[12] L. H. S. Cevidanes, A.-K. Hajati, B. Paniagua et al., "Quantification of condylar resorption in temporomandibular joint osteoarthritis," Oral Surgery, Oral Medicine, Oral Pathology, Oral Radiology, and Endodontology, vol. 110, no. 1, pp. 110$117,2010$.

[13] M. O. Lagravère, L. Hansen, W. Harzer, and P. W. Major, "Plane orientation for standardization in 3-dimensional cephalometric analysis with computerized tomography imaging," American Journal of Orthodontics and Dentofacial Orthopedics, vol. 129, no. 5, pp. 601-604, 2006.

[14] R. Leonardi, S. Muraglie, A. Lo Giudice, K. S. Aboulazm, and R. Nucera, "Evaluation of mandibular symmetry and morphology in adult patients with unilateral posterior crossbite: a CBCT study using a surface-to-surface matching technique," European Journal of Orthodontics, vol. 42, no. 6, p. 650, 2020.

[15] S. Muraglie, R. Leonardi, K. Aboulazm, C. Stumpo, C. Loreto, and C. Grippaudo, "Evaluation of structural skeletal asymmetry of the glenoid fossa in adult patients with unilateral posterior crossbite using surface-to-surface matching on CBCT images," The Angle Orthodontist, vol. 90, no. 3, pp. 376-382, 2020.

[16] R. Leonardi, S. Muraglie, O. Bennici, C. Cavallini, and C. Spampinato, "Three-dimensional analysis of mandibular functional units in adult patients with unilateral posterior crossbite: a cone beam study with the use of mirroring and surface-to-surface matching techniques," The Angle Orthodontist, vol. 89, no. 4, pp. 590-596, 2019.

[17] A. C. Ruellas, M. S. Yatabe, B. Q. Souki et al., "3D mandibular superimposition: comparison of regions of reference for voxel-based registration," PLoS One, vol. 11, Article ID e0157625, 2016.

[18] L. H. S. Cevidanes, L. T. J. Bailey, S. F. Tucker et al., "Threedimensional cone-beam computed tomography for assessment of mandibular changes after orthognathic surgery," American Journal of Orthodontics and Dentofacial Orthopedics, vol. 131, no. 1, pp. 44-50, 2007.

[19] L. H. S. Cevidanes, M. A. Styner, and W. R. Proffit, "Image analysis and superimposition of 3-dimensional cone-beam computed tomography models," American Journal of Orthodontics and Dentofacial Orthopedics, vol. 129, no. 5, pp. 611-618, 2006.

[20] T. Nguyen, L. Cevidanes, L. Franchi, A. Ruellas, and T. Jackson, "Three-dimensional mandibular regional superimposition in growing patients," American Journal of Orthodontics and Dentofacial Orthopedics, vol. 153, no. 5, pp. 747-754, 2018.

[21] S. Stucki and N. Gkantidis, "Assessment of techniques used for superimposition of maxillary and mandibular 3D surface models to evaluate tooth movement: a systematic review," European Journal of Orthodontics, vol. 42, no. 5, pp. 559-570, 2019.
[22] L. Koerich, D. Burns, A. Weissheimer, and J. D. P. Claus, "Three-dimensional maxillary and mandibular regional superimposition using cone beam computed tomography: a validation study," International Journal of Oral and Maxillofacial Surgery, vol. 45, no. 5, pp. 662-669, 2016.

[23] M. G. Hans, J. M. Palomo, and M. Valiathan, "History of imaging in orthodontics from Broadbent to cone-beam computed tomography," American Journal of Orthodontics and Dentofacial Orthopedics, vol. 148, no. 6, pp. 914-921, 2015.

[24] B. Melsen, "Time of closure of the spheno-occipital synchondrosis determined on dry skulls A radiographic craniometric study," Acta Odontologica Scandinavica, vol. 27, no. 1-2, pp. 73-90, 1969.

[25] P.-G. Planché, "La méthode structurale de Arne Björk et Vibeke Skieller," L'Orthodontie Française, vol. 77, no. 3, pp. 347-358, 2006.

[26] B. H. Broadbent, "Bolton standards and technique in orthodontic practice," The Angle Orthodontist, vol. 7, pp. 209233, 1937.

[27] A. W. Moore, "Orthodontic treatment factors in Class II malocclusion," American Journal of Orthodontics and Dentofacial Orthopedics, vol. 45, pp. 323-352, 1959.

[28] A. Weissheimer, L. M. Menezes, L. Koerich, J. Pham, and L. H. S. Cevidanes, "Fast three-dimensional superimposition of cone beam computed tomography for orthopaedics and orthognathic surgery evaluation," International Journal of Oral and Maxillofacial Surgery, vol. 44, no. 9, pp. 1188-1196, 2015.

[29] R. M. Ricketts, "The influence of orthodontic treatment on facial growth and development," The Angle Orthodontist, vol. 30, pp. 103-132, 1960.

[30] J. A. McNamara Jr., "A method of cephalometric evaluation," American Journal of Orthodontics, vol. 86, no. 6, pp. 449-469, 1984.

[31] W. B. Downs, "Variations in facial relationships: their significance in treatment and prognosis," American Journal of Orthodontics, vol. 34, no. 10, pp. 812-840, 1948.

[32] A. G. Brodie, "Cephalometric roentgenology: history, technics and uses," Journal of Oral Surgery, vol. 7, pp. 185-198, 1949.

[33] R. A. Riedel, "A postretention evaluation," The Angle Orthodontist, vol. 44, pp. 194-212, 1974.

[34] S. E. Coben, Basion Horizontal: An integrated. Concept of Craniofacial Growth and Cephalometric Analysis, Computer Cephalometric Associated, Jenkintown, PA, USA, 1986.

[35] A. Björk and V. Skieller, "Postnatal growth and development of the maxillary complex," in Factors Affecting the Growth of the Midface, J. A. McNamara, Ed., pp. 61-99, University of Michigan, Ann Arbor, MI, USA, 1976.

[36] M. Y. Hajeer, A. F. Ayoub, and D. T. Millett, "Three-dimensional assessment of facial soft-tissue asymmetry before and after orthognathic surgery," British Journal of Oral and Maxillofacial Surgery, vol. 42, no. 5, pp. 396-404, 2004.

[37] R. Leonardi, S. Muraglie, S. Crimi, M. Pirroni, G. Musumeci, and R. Perrotta, "Morphology of palatally displaced canines and adjacent teeth, a 3-D evaluation from cone-beam computed tomographic images," BMC Oral Health, vol. 18, no. 1, p. 156, 2018.

[38] L. Cevidanes, L. Bailey, G. Tucker Jr. et al., "Superimposition of 3D cone-beam CT models of orthognathic surgery patients," Dentomaxillofacial Radiology, vol. 34, no. 6, pp. 369-375, 2005.

[39] G. Han, J. Li, S. Wang, Y. Liu, X. Wang, and Y. Zhou, "In-vitro assessment of the accuracy and reliability of mandibular dental model superimposition based on voxel-based cone- 
beam computed tomography registration," The Korean Journal of Orthodontics, vol. 49, no. 2, pp. 97-105, 2019.

[40] T. Nguyen, L. Cevidanes, M. A. Cornelis, G. Heymann, L. K. de Paula, and H. De Clerck, "Three-dimensional assessment of maxillary changes associated with bone anchored maxillary protraction," American Journal of Orthodontics and Dentofacial Orthopedics, vol. 140, no. 6, pp. 790-798, 2011.

[41] J. A. Salzmann, "The research workshop on cephalometrics," American Journal of Orthodontics, vol. 46, pp. 834-847, 1960.

[42] R. M. Nada, T. J. Maal, K. H. Breuning, S. J. Berge, Y. A. Mostafa, and A. M. Kuijpers-Jagtman, "Accuracy and reproducibility of voxel based superimposition of cone beam computed tomography models on the anterior cranial base and the zygomatic arches," PLoS One, vol. 6, Article ID e16520, 2011.

[43] L. H. C. Cevidanes, G. Heymann, M. A. Cornelis, H. J. DeClerck, and J. F. C. Tulloch, "Superimposition of 3dimensional cone-beam computed tomography models of growing patients," American Journal of Orthodontics and Dentofacial Orthopedics, vol. 136, no. 1, pp. 94-99, 2009.

[44] J. Schilling, L. C. Gomes, E. Benavides et al., "Regional 3D superimposition to assess temporomandibular joint condylar morphology," Dentomaxillofacial Radiology, vol. 43, Article ID 20130273, 2014.

[45] A. Almukhtar, X. Ju, B. Khambay, J. McDonald, and A. Ayoub, "Comparison of the accuracy of voxel based registration and surface based registration for 3D assessment of surgical change following orthognathic surgery," PLoS One, vol. 9, Article ID e93402, 2014. 